\title{
KSP: A Resolution-based Prover for Multimodal K Abridged Report
}

\author{
Cláudia Nalon \\ Department of Computer Science \\ University of Brasília \\ nalon@unb.br
}

\begin{abstract}
We briefly describe an implementation of a hyperresolution-based calculus for the propositional basic multimodal logic, $\mathrm{K}_{n}$. The prover, $\mathrm{K}_{\mathrm{S}} \mathrm{P}$, which allows for both local and global reasoning, is designed to support experimentation with different combinations of refinements for its basic calculus. We present an experimental evaluation that compares $\mathrm{KS}_{S} \mathrm{P}$ with a range of existing reasoners for $\mathrm{K}_{n}$.
\end{abstract}

\section{Introduction}

Modal logics have long been used in Computer Science for describing and reasoning about complex systems, including programming languages [Pratt, 1980], knowledge representation and reasoning [Rao and Georgeff, 1991; Halpern and Moses, 1992], verification of distributed systems [Hailpern, 1982; Halpern et al., 1983; Halpern, 1987] and terminological reasoning [Schild, 1991]. The most basic of such logics is the multimodal $\mathrm{K}_{n}$, which extends the classical language with new operators, $a$ and $\hat{y}$, with $a \in \mathrm{A}=\{1, \ldots, n\}$, a fixed finite set of indexes (Section 2). The local satisfiability problem for the multimodal propositional case is PSPACEcomplete [Halpern and Moses, 1992]. The global satisfiability and the local satisfiability under global constraint problems for $\mathrm{K}_{n}$ are EXPTIME-complete [Spaan, 1993]. Given the inherent intractability of the reasoning problem and also the wide range of applications to which those logics can be applied, the development of automatic, efficient tools for theorem proving is highly desirable.

Several proof methods and tools for modal reasoning exist, either in the form of methods applied direct to the modal language or obtained by translation into more expressive languages (First-Order Logic, for instance). Here we concentrate on a direct method for $\mathrm{K}_{n}$, the modal resolution procedure described in [Nalon et al., 2015]. Resolution [Robinson, 1965] is a saturation procedure, that is, formulae which are consequence of a given input set are added to this set until a contradiction is derived or the satisfiability of the set is evident. Following successful refinements and strategies used in theorem-provers for classical logic (see [Schulz and Möhrmann, 2016], for instance), our calculus is designed for restricting as much as possible the number of clauses produced during saturation. In particular, we take advantage of

\author{
Ullrich Hustadt and Clare Dixon \\ Department of Computer Science \\ University of Liverpool \\ \{U.Hustadt,C.Dixon\}@liverpool.ac.uk
}

well-known properties for the satisfiability problem for $\mathrm{K}_{n}$, namely: a formula $\varphi$ is satisfiable if, and only if, it is satisfiable in a finite tree-like model; and also that checking the satisfiability of a subformula associated with level $\mathrm{ml}$ of the tree depends only on the subformulae occurring at levels greater or equal to $m l$ [Areces et al., 2000]. The procedure, which is presented in Section 3, requires the translation of a formula $\varphi$ into a more expressive language, where labels are used to denote the level at which $\varphi$ occurs. Labelled resolution is then applied in order to determine the satisfiability of $\varphi$.

In this paper, we briefly present $\mathrm{K}_{S} \mathrm{P}$, a theorem prover for the basic multimodal logic $\mathrm{K}_{n}$ which implements the calculus described in [Nalon et al., 2015] as a variation of the set of support strategy [Wos et al., 1965]. The prover also implements several other refinements and simplification techniques in order to reduce the search space for a proof (Section 4). Besides the set of support strategy, all other refinements of the calculus are implemented as independent modules, allowing for a better evaluation of how effective they are. The experimental evaluation indicates that $\mathrm{K}_{\mathrm{S}} \mathrm{P}$ works well on problems with high nesting of modal operators where the separation of modal layers can be exploited to improve the efficiency of reasoning (Section 5). An extended version of this paper can be found at [Nalon et al., 2016a].

\section{Language}

Let $\mathrm{A}=\{1, \ldots, n\}, n \in \mathbb{N}$, be a finite fixed set of indexes and $\mathrm{P}=\left\{p, q, s, t, p^{\prime}, q^{\prime}, \ldots\right\}$ be a denumerable set of propositional symbols. The set of modal formulae, WFF, is the least set such that every $p \in \mathrm{P}$ is in WFF; if $\varphi$ and $\psi$ are in WFF, then so are $\neg \varphi,(\varphi \wedge \psi)$, and $\square \varphi$ for each $a \in \mathrm{A}$. The formulae false, true, $(\varphi \vee \psi),(\varphi \Rightarrow \psi)$, and $\hat{\Delta} \varphi$ are introduced as the abbreviations for $(\varphi \wedge \neg \varphi), \neg$ false, $\neg(\neg \varphi \wedge \neg \psi)$, $(\neg \varphi \vee \psi)$, and $\neg \square \neg \varphi$, respectively (where $\varphi, \psi \in$ WFF). A literal is either a propositional symbol or its negation; the set of literals is denoted by L. A modal literal is either $a l$ or a $l$, where $l \in \mathrm{L}$ and $a \in \mathrm{A}$. The literals (resp. modal literals) $l$ and $\neg l$ (resp. $\square l$ and $\hat{\Delta} \neg l=\neg \square l$ ) are said to be complementary. The modal depth of a formula is given by the maximal number of nested occurrences of modal operators in that formula. The modal level of a subformula is the maximal number of nested occurrences of modal operators in which scope the formula occurs. For instance, in $\square[\hat{a} p$, 
Proceedings of the Twenty-Sixth International Joint Conference on Artificial Intelligence (IJCAI-17)

$$
\begin{aligned}
& \text { [LRES] } m l_{1}: \quad D \vee l \quad \text { [MRES] } m l_{1}: l_{1} \Rightarrow a l
\end{aligned}
$$

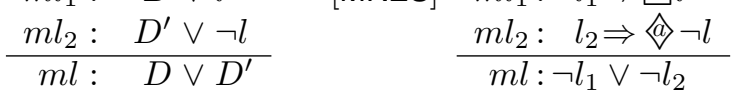

$$
\begin{aligned}
& \text { [GEN2] } m l_{1}: l_{1}^{\prime} \Rightarrow a l_{1} \\
& m l_{2}: l_{2}^{\prime} \Rightarrow a \neg l_{1} \\
& \frac{m l_{3}: l_{3}^{\prime} \Rightarrow \Delta l_{2}}{m l: \neg l_{1}^{\prime} \vee \neg l_{2}^{\prime} \vee \neg l_{3}^{\prime}} \\
& \text { [GEN1] } m l_{1}: l_{1}^{\prime} \Rightarrow a \neg l_{1} \\
& m l_{m}: l_{m}^{\prime} \stackrel{\vdots}{\Rightarrow} \neg l_{m} \\
& m l_{m+1}: l^{\prime} \Rightarrow\langle\hat{\Delta} \neg l \\
& \frac{m l_{m+2}: l_{1} \vee \ldots \vee l_{m} \vee l}{m l: \neg l_{1}^{\prime} \vee \ldots \vee \neg l_{m}^{\prime} \vee \neg l^{\prime}} \\
& m l_{1}: l_{1}^{\prime} \Rightarrow a \neg l_{1} \\
& m l_{m}: l_{m}^{\prime} \stackrel{\vdots}{\Rightarrow} \neg l_{m} \\
& m l_{m+1}: l^{\prime} \Rightarrow\langle\hat{a} l \\
& \frac{m l_{m+2}: l_{1} \vee \ldots \vee l_{m}}{m l: \neg l_{1}^{\prime} \vee \ldots \vee \neg l_{m}^{\prime} \vee \neg l^{\prime}}
\end{aligned}
$$

Figure 1: Inference rules, where $m l=\sigma\left(\left\{m l_{1}, \ldots, m l_{m+1}, m l_{m+2}-1\right\}\right)$ in GEN1, GEN3; $m l=\sigma\left(\left\{m l_{1}, m l_{2}\right\}\right)$ in LRES, MRES; and $m l=\sigma\left(\left\{m l_{1}, m l_{2}, m l_{3}\right\}\right)$ in GEN2.

the modal depth of $p$ is 0 and its modal level is 2 . Formal definitions can be found in [Nalon et al., 2015].

As our calculus operates on a labelled clausal normal form that is closely linked to the tree model property of Kripke models for $\mathrm{K}_{n}$, we briefly state the semantics of $\mathrm{K}_{n}$. A treelike Kripke model $M$ for $n$ agents over $\mathrm{P}$ is given by a tuple $\left(W, w_{0}, R_{1}, \ldots, R_{n}, \pi\right)$, where $W$ is a set of possible worlds with a distinguished world $w_{0}$, each accessibility relation $R_{a}$ is a binary relation on $W$ such that their union is a tree with root $w_{0}$, and $\pi: W \rightarrow(\mathrm{P} \rightarrow\{$ true,false $\})$ is a function which associates with each world $w \in W$ a truthassignment to propositional symbols. Satisfaction of a formula at a world $w$ of a model $M$ is defined by:

- $\langle M, w\rangle \models p$ iff $\pi(w)(p)=$ true, where $p \in \mathrm{P}$;

- $\langle M, w\rangle \models \neg \varphi$ iff $\langle M, w\rangle \not \models \varphi$;

- $\langle M, w\rangle \models(\varphi \wedge \psi)$ iff $\langle M, w\rangle \models \varphi$ and $\langle M, w\rangle \models \psi$;

- $\langle M, w\rangle \models \square \varphi$ iff for all $w^{\prime}$, if $w R_{a} w^{\prime}$ then $\left\langle M, w^{\prime}\right\rangle \models \varphi$. Let $M=\left(W, w_{0}, R_{1}, \ldots, R_{n}, \pi\right)$ be a model. A formula $\varphi$ is locally satisfied in $M$, denoted by $M \models_{L} \varphi$, if $\left\langle M, w_{0}\right\rangle \models \varphi$. The formula $\varphi$ is locally satisfiable if there is a model $M$ such that $\left\langle M, w_{0}\right\rangle=\varphi$. A formula $\varphi$ is globally satisfied in $M$, if for all $w \in W,\langle M, w\rangle \models \varphi$. We denote by $\operatorname{depth}(w)$ the length of the unique path from $w_{0}$ to $w$ through the union of the accessibility relations in $M$. We call a modal layer the equivalence class of worlds at the same depth in a model.

We note that checking the local satisfiability of a formula $\varphi$ can be reduced to the problem of checking the local satisfiability of its subformulae at the modal layer of a model which corresponds to the modal level where those subformulae occur (see [Areces et al., 2000]). Also, checking the global satisfiability of $\varphi$ can be reduced to checking the local satisfiability of $\varphi$ at all modal layers (up to an exponential distance from the root) of a model [Goranko and Passy, 1992; Spaan, 1993]. Thus, an uniform approach based on modal levels can be used to deal with both problems, as given in the next section.

\section{A Normal Form and Calculus for $\mathrm{K}_{n}$}

The calculus operates on labelled clauses of the form $m l: C$ (literal clause), $m l: l \Rightarrow \square l^{\prime}$ (positive $a$-clause), $m l: l \Rightarrow$ (a) $l^{\prime}$ (negative $a$-clause) where $m l \in \mathbb{N} \cup\{*\}, C$ is a propositional clause, and $l, l^{\prime}$ are literals. The label $m l$ indicates the depth of the Kripke model at which the clause is true. The special label $*$ is used if a clause is true at all depths/worlds in a model and it normally only occurs in the normal form if we want to check a formula for global satisfiability. Any formula of $\mathrm{K}_{n}$ can be transformed into an equi-satisfiable set of labelled clauses. The calculus uses a simple form of unification. The partial unification function $\sigma$ on sets of labels is given by $\sigma(\{m l, *\})=m l$; and $\sigma(\{m l\})=m l$; otherwise, $\sigma$ is undefined. The inference rules (Figure 1) can only be applied if the unification on labels is defined.

We show next an example of a refutation which uses both local and global reasoning (from [Nalon et al., 2015], adapted from [Areces et al., 1999]). Clauses 1 and 2 say that a person is either female or male. Clauses 3 and 4 say that tall people have children with blond hair. The particular situation of Tom, denoted here by $t_{0}$, is given in the following clauses. Clauses 5, 6, and 7 say that Tom's daughters are tall. Clauses 8 and 9 say that Tom has a grandchild who is not blond. We want to prove that Tom has a son, which appears negated in Clause 10. The refutation is given below.
1. * : female $\vee$ male
2. * : $\neg$ female $\vee \neg$ male
3. $*$ : $\neg$ tall $\vee t_{1}$
4. *: $t_{1} \Rightarrow$ c blond
5. $0: t_{0}$
6. $0: t_{0} \Rightarrow c t_{2}$
7. $1: \neg t_{2} \vee \neg$ female $\vee$ tall
8. $0: t_{0} \Rightarrow \diamond t_{3}$
9. $1: t_{3} \Rightarrow \widehat{\diamond} \neg$ blond
10. $0: t_{0} \Rightarrow \emptyset \neg$ male
11. $1: \neg t_{1} \vee \neg t_{3}$
12. $1: \neg$ tall $\vee \neg t_{3}$
13. $1: \neg t_{3} \vee \neg t_{2} \vee \neg$ female
14. 1 : male $\vee \neg t_{2} \vee \neg t_{3}$
15. $0: \neg t_{0}$
16. 0 : false

\author{
[MRES, 9, 4] \\ [LRES, 11,3 ] \\ [LRES, 7,12$]$ \\ [LRES, 13, 1] \\ [GEN1, 10, 6, 8, 14]
}
[LRES, 15, 5]

\section{$4 \mathrm{KS}_{\mathrm{S}}$}

$\mathrm{K}_{\mathrm{S}} \mathrm{P}$ is an implementation, written in $\mathrm{C}$, of the calculus in Figure 1. The main loop is based on the given-clause algorithm implemented in Otter [McCune, 2007], a variation of the set of support strategy [Wos et al., 1965], a refinement 


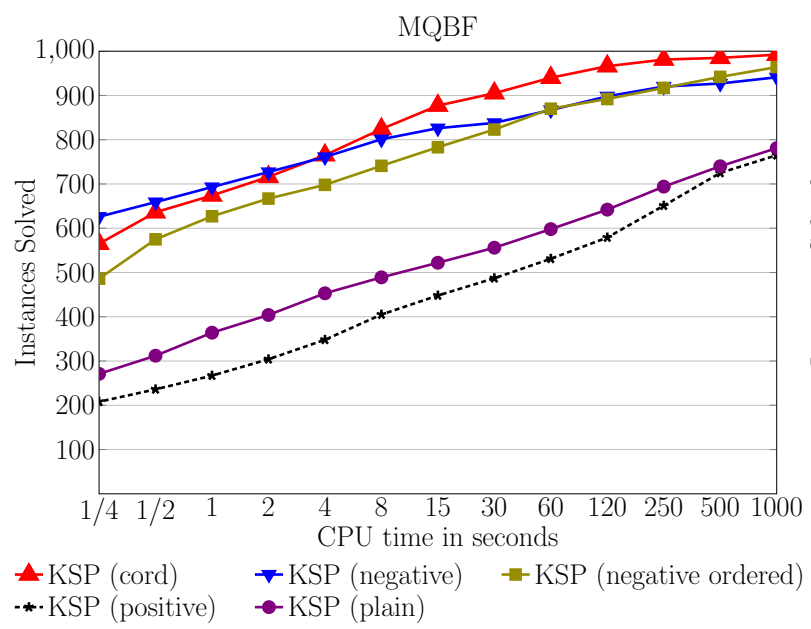

(a) $\mathrm{KS}_{\mathrm{S}} \mathrm{P}$ with different refinements

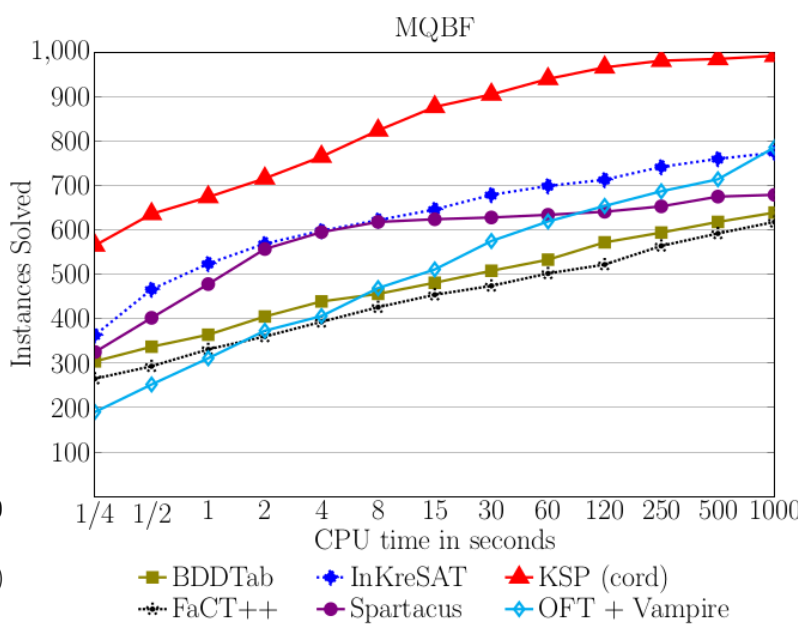

(b) All provers

Figure 2: Benchmarking results for MQBF

which restricts the set of choices of clauses participating in a derivation step. For the modal calculus, the set of clauses is partitioned according to the modal layer at which clauses are true. For each modal level $m l$, a literal clause is selected and the prover tries to resolve it with either a literal clause at the same modal level or with a set of modal clauses in the previous modal level, $m l-1$.

The partitioning of the set of clauses avoids unnecessary applications of the resolution inference rules. For instance, the translation of the formula $\varphi=\widehat{\Delta}\langle\vec{\Delta} p \wedge a \neg p$ without considering the modal level at which its subformulae occur results in the set:

$$
\begin{array}{ll}
\text { 1. } & t_{0} \\
\text { 2. } & t_{0} \Rightarrow \triangleq t_{1} \\
\text { 3. } & t_{1} \Rightarrow \triangleq \overrightarrow{ } \\
\text { 4. } & t_{0} \Rightarrow a \neg p
\end{array}
$$

where $t_{0}$ and $t_{1}$ are new propositional symbols. Because $\langle\hat{\Delta} p$ and $a \neg p$ are complementary, resolution applied to Clauses 3 and 4 results in $\neg t_{0} \vee \neg t_{1}$, which cannot be used to find a contradiction. In contrast, by using labels, the translation of $\varphi$ results in the set:

$$
\begin{array}{lll}
1^{\prime} . & 0: & t_{0} \\
2^{\prime} . & 0: & t_{0} \Rightarrow \Delta \Delta t_{1} \\
3^{\prime} . & 1: & t_{1} \Rightarrow \Delta \Delta p \\
4^{\prime} . & 0: & t_{0} \Rightarrow \Delta \neg p
\end{array}
$$

As the labels indicate, Clauses $3^{\prime}$ and $4^{\prime}$ occur at different modal levels and resolution is not applied to those clauses.

As another simple example, consider the formula $a \square p \wedge$ \a $\langle\vec{\Delta} \neg p$, whose layered normal form is given below.

$$
\begin{aligned}
& \text { 1. } 0: t_{0} \\
& \text { 2. } 0: t_{0} \Rightarrow a t_{1} \\
& \text { 3. } 1: t_{1} \Rightarrow a p \\
& \text { 4. } 0: t_{0} \Rightarrow \triangleq t_{2} \\
& \text { 5. } \left.1: t_{2} \Rightarrow \widehat{a}\right\rangle \neg p
\end{aligned}
$$

As clauses 3 and 4 are at the same modal level, MRES can be applied, resulting in $0: \neg t_{1} \vee \neg t_{2}$. By an application of GEN1 to this clause and Clauses 2 and 4, we obtain $0: \neg t_{0}$. Applying LRES to $0: \neg t_{0}$ and Clause 1 , results in 0 : false, which shows that the original formula is unsatisfiable.

Besides the basic calculus with a set-of-support strategy, the user can further restrict LRES by choosing ordered (clauses can only be resolved on their maximal literals with respect to an ordering chosen by the prover in such a way to preserve completeness), negative (one of the premises is a negative clause, i.e. a clause where all literals are of the form $\neg p$ for some $p \in \mathrm{P}$ ), positive (one of the premises is a positive clause), or negative + ordered resolution (both negative and ordered resolution inferences are performed). The completeness of some of these refinements depends on the particular normal form chosen. The prover can either perform local or global reasoning. For a comprehensive description of $\mathrm{KS}_{\mathrm{S}} \mathrm{P}$ see [Nalon et al., 2016a; Nalon et al., 2016b].

\section{Evaluation}

We have compared $\mathrm{K}_{\mathrm{S}} \mathrm{P}$ with BDDTab [Goré et al., 2014], FaCT++ 1.6.3 [Tsarkov and Horrocks, 2006], InKreSAT 1.0 [Kaminski and Tebbi, 2013], Spartacus 1.0 [Götzmann et al., 2010], and a combination of the optimised functional translation [Horrocks et al., 2006] with Vampire 3.0 [Kovács and Voronkov, 2013]. Benchmarking was performed on PCs with an Intel i7-2600 CPU @3.40GHz and 16GB main memory. For each formula and each prover we have determined the median run time over five runs with a time limit of $1000 \mathrm{CPU}$ seconds for each run. The experiments are fully described in [Nalon et al., 2016a], from where the graphs shown in this section are taken.

Our benchmarks [Nalon et al., 2016b] consist of three collections of modal formulae:

1. The complete set of TANCS-2000 modalised random QBF (MQBF) formulae [Massacci and Donini, 2000] complemented by the additional MQBF formulae provided by 


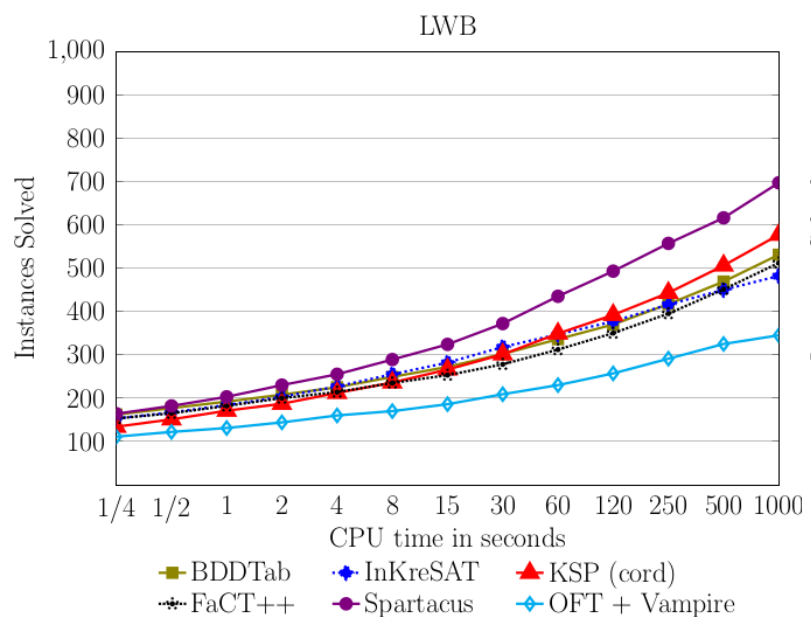

(a) Results for LWB

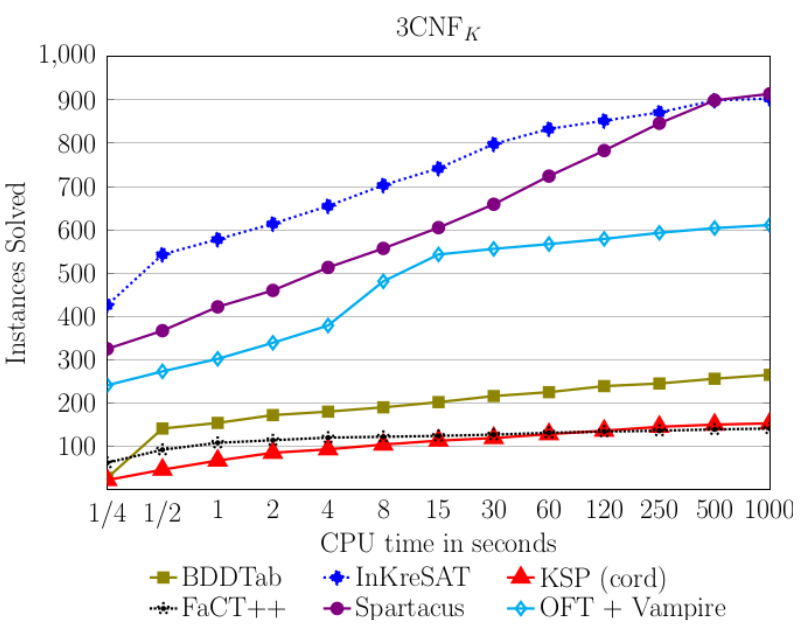

(b) Results for $3 \mathrm{CNF}_{K}$

Figure 3: Benchmarking results for $\mathrm{LWB}$ and $3 \mathrm{CNF}_{\mathrm{K}}$

Tebbi and Kaminski [2013]. This collection consists of five classes, with a total of 1016 formulae, of which 617 are known to be satisfiable and 399 are known to be unsatisfiable (due to the output of one of the provers).

2. Logics Workbench (LWB) basic modal logic benchmark formulae [Balsiger et al., 2000], with 56 formulae chosen from each of the 18 parameterised classes. The median value of the maximal parameter value used for the 18 classes is 1880 , far beyond what has ever been tested before. Of the resulting 1008 formulae, half are satisfiable and half are unsatisfiable by construction of the benchmark classes.

3. Randomly generated $3 \mathrm{CNF}_{\mathrm{K}}$ formulae [Patel-Schneider and Sebastiani, 2003] over 3 to 10 propositional symbols with modal depth 1 or 2 . The formulae were chosen from each of the 11 parameter settings given in [Patel-Schneider and Sebastiani, 2003, page 372]. The resulting collection contains 1000 formulae, of which 457 are known to be satisfiable and 464 are known to be unsatisfiable.

Figure 2a compares the impact of different refinements on the performance of $\mathrm{KS}_{S} \mathrm{P}$ on the MQBF collection. With plain $\mathrm{KS}_{\mathrm{S}} \mathrm{P}$ uses the rules shown in Figure 1, without additional refinement. With cord and negative ordered, $\mathrm{K}_{\mathrm{SP}}$ applies ordered resolution and negative + ordered resolution, respectively. The configuration negative (resp. positive) uses negative (resp. positive) resolution on a set of clauses. For all configurations, the same preprocessing techniques are used. The cord configuration offers the best performance. Ordered resolution restricts the applicability of the rules further than the other refinements. Not only is this an advantage on satisfiable formulae in that a saturation can be found more quickly, but also on unsatisfiable formulae where with this refinement $\mathrm{K}_{\mathrm{S}} \mathrm{P}$ finds refutations much more quickly than with any of the other refinements.

Figure $2 \mathrm{~b}$ compares the performance of all the provers on the MQBF collection. It shows that $\mathrm{K}_{\mathrm{S}} \mathrm{P}$ performs better than any of the other provers. The classes of formulae on which $\mathrm{KS}_{\mathrm{S}} \mathrm{P}$ performs best are those where the number of proposi- tional symbols is small and/or those symbols are uniformly distributed over a wide range of modal levels. This reduces the possibility of inference steps between modal clauses with complementary literals.

Figure 3 shows the benchmarking results on the LWB and $3 \mathrm{CNF}_{\mathrm{K}}$ collections. On the LWB collection $\mathrm{K}_{\mathrm{S}} \mathrm{P}$ performs about as well as BDDTab, FaCT++ and InKreSAT, while Spartacus performs best and the combination of the optimised functional translation with Vampire (OFT + Vampire) performs worst. A characteristic of the classes on which $\mathrm{KS}_{\mathrm{S}} \mathrm{P}$ performs best is again that atomic subformulae are evenly spread over a wide range of modal levels.

Finally, on the $3 \mathrm{CNF}_{\mathrm{K}}$ collection, InKreSAT is the best performing prover and $\mathrm{K}_{\mathrm{S}} \mathrm{P}$ the worst one. This should now not come as a surprise. For $3 \mathrm{CNF}_{\mathrm{K}}$ we specifically restricted ourselves to formulae with low modal depth which in turn means that the layered normal form has little positive effect.

\section{Conclusions and Future Work}

$\mathrm{K}_{\mathrm{S}} \mathrm{P}$ implements a variation of the set-of-support strategy by restricting further the application of inference rules to clauses whose literals are at the same modal level. The evaluation indicates that $\mathrm{K}_{\mathrm{S}} \mathrm{P}$ works well on problems with high modal depth where the separation of modal layers can be exploited to improve the efficiency of reasoning.

The prover implements both local and global reasoning, and the implementation of the local satisfiability under global constraint procedure is ongoing work. We are are also working on the extension of the calculus for dealing with different modal logics. The calculi for $\mathrm{T}_{n}$ and $4_{n}$, which can be combined to provide a calculus for $\mathrm{S} 4_{n}$, have already been implemented.

Several refinements and redundancy elimination techniques have been implemented. The choice of strategies and optimisations are, by now, all left to the user. The development of an "auto mode" in which the prover makes a choice of its own, based on an analysis of the given formula, is left for future work. 


\section{References}

[Areces et al., 1999] Carlos Areces, Hans de Nivelle, and Maarten de Rijke. Prefixed Resolution: A Resolution Method for Modal and Description Logics. In Proc. of CADE-16, volume 1632 of LNAI, pages 187-201. Springer, 1999.

[Areces et al., 2000] Carlos Areces, Rosella Gennari, Juan Heguiabehere, and Maarten de Rijke. Tree-based heuristics in modal theorem proving. In Proc. of ECAI 2000, pages 199-203. IOS Press, 2000.

[Balsiger et al., 2000] Peter Balsiger, Alain Heuerding, and Stefan Schwendimann. A benchmark method for the propositional modal logics K, KT, S4. Journal of Automated Reasoning, 24(3):297-317, 2000.

[Goranko and Passy, 1992] Valentin Goranko and Solomon Passy. Using the universal modality: gains and questions. Journal of Logic and Computation, 2(1):5-30, 1992.

[Goré et al., 2014] Rajeev Goré, Kerry Olesen, and Jimmy Thomson. Implementing tableau calculi using BDDs: BDDTab system description. In Proc. of IJCAR 2014, volume 8562 of $L N C S$, pages 337-343. Springer, 2014.

[Götzmann et al., 2010] Daniel Götzmann, Mark Kaminski, and Gert Smolka. Spartacus: A tableau prover for hybrid logic. ENTCS, 262:127-139, 2010.

[Hailpern, 1982] Brent T. Hailpern. Verifying Concurrent Processes Using Temporal Logic, volume 129 of LNCS. Springer, 1982.

[Halpern and Moses, 1992] Joseph Y. Halpern and Yoram Moses. A guide to completeness and complexity for modal logics of knowledge and belief. Artificial Intelligence, 54(3):319-379, April 1992.

[Halpern et al., 1983] Joseph Y. Halpern, Zohar Manna, and Ben Moszkowski. A Hardware Semantics Based on Temporal Intervals. In Proc. of ICALP 1983, volume 154, pages 278-291, 1983.

[Halpern, 1987] Joseph Y. Halpern. Using Reasoning about Knowledge to Analyze Distributed Systems. Annual Review of Computer Science, 2, 1987.

[Horrocks et al., 2006] Ian R. Horrocks, Ullrich Hustadt, Ulrike Sattler, and Renate Schmidt. Computational modal logic. In Handbook of Modal Logic, pages 181-245. Elsevier, 2006.

[Kaminski and Tebbi, 2013] Mark Kaminski and Tobias Tebbi. InKreSAT: Modal reasoning via incremental reduction to SAT. In Proc. of CADE-24, volume 7898 of LNAI, pages 436-442. Springer, 2013.

[Kovács and Voronkov, 2013] Laura Kovács and Andrei Voronkov. First-order theorem proving and Vampire. In Proc. of CAV 2013, volume 8044 of LNCS, pages 1-35. Springer, 2013.

[Massacci and Donini, 2000] Fabio Massacci and Francesco M. Donini. Design and results of TANCS-2000 non-classical (modal) systems comparison. In Proc. of TABLEAUX 2000, volume 1847 of LNCS, pages 52-56. Springer, 2000.
[McCune, 2007] William W. McCune. OTTER 3.0 reference manual and guide, May 072007.

[Nalon et al., 2015] Cláudia Nalon, Ullrich Hustadt, and Clare Dixon. A modal-layered resolution calculus for $\mathrm{K}$. In Proc. of TABLEAUX 2015, volume 9323 of LNCS, pages 185-200. Springer, 2015.

[Nalon et al., 2016a] Cláudia Nalon, Ullrich Hustadt, and Clare Dixon. $\mathrm{KS}_{\mathrm{S}} \mathrm{P}$ : A resolution-based prover for multimodal K. In Proc. of IJCAR 2016, volume 9706 of LNCS, pages 406-415. Springer, 2016.

[Nalon et al., 2016b] Cláudia Nalon, Ullrich Hustadt, and Clare Dixon. $\mathrm{KS}_{\mathrm{S}} \mathrm{P}$ : sources and benchmarks. http: //www. cic.unb.br/ nalon/\# software, 2016.

[Patel-Schneider and Sebastiani, 2003] Peter F. PatelSchneider and Roberto Sebastiani. A new general method to generate random modal formulae for testing decision procedures. Journal of Artificial Intelligence Research (JAIR), 18:351-389, 2003.

[Pratt, 1980] Vaughan R. Pratt. Application of modal logic to programming. Studia Logica, 39(2/3):257-274, 1980.

[Rao and Georgeff, 1991] Anand S. Rao and Michael P. Georgeff. Modeling Rational Agents within a BDIArchitecture. In Proc. of KR\&R-91, pages 473-484, Cambridge, MA, USA, April 1991. Morgan-Kaufmann.

[Robinson, 1965] John A. Robinson. A Machine-Oriented Logic Based on the Resolution Principle. Journal of the ACM, 12(1):23-41, January 1965.

[Schild, 1991] Klaus Schild. A Correspondence Theory for Terminological Logics. In Proc. of IJCAI'91, pages 466471. Springer, 1991.

[Schulz and Möhrmann, 2016] Stephan Schulz and Martin Möhrmann. Performance of clause selection heuristics for saturation-based theorem proving. In Proc. of IJCAR 2016, volume 9706 of $L N C S$, pages 330-345. Springer, 2016.

[Spaan, 1993] Edith Spaan. Complexity of Modal Logics. PhD thesis, University of Amsterdam, 1993.

[Tsarkov and Horrocks, 2006] Dmitry Tsarkov and Ian Horrocks. FaCT++ description logic reasoner: System description. In Proc. of IJCAR 2006, volume 4130 of LNCS, pages 292-297. Springer, 2006.

[Wos et al., 1965] Lawrence Wos, George A. Robinson, and Daniel F. Carson. Efficiency and Completeness of the Set of Support Strategy in Theorem Proving. Journal of the ACM, 12:536-541, 1965. 